\title{
Role of a Neonatal Intensive Care Unit during the COVID-19 Pandemia: recommendations from the neonatology discipline
}

\author{
(iD) Werther Brunow de Carvalho' \\ DMaria Auqusta Bento Cicaroni Gibelli² \\ (iD) Vera Lucia Jornada Krebs ${ }^{3}$ \\ (iDCarla Regina Tragante ${ }^{2}$ \\ (iD) Maria Beatriz Moliterno Perondi ${ }^{4}$
}

\begin{abstract}
1. Terapia Intensiva em Neonatologia/Pediatria, Departamento de Pediatria, Faculdade de Medicina (FMUSP), Universidade de São Paulo, SP, Brasil. 2. Centro Neonatal e Terapia Intensiva, Instituto da Crianca e do Adolescente (ICr), Hospital das Clínicas HCFMUSP, Faculdade de Medicina, Universidade de São Paulo, SP, Brasil.

3. Curso de Pos-Graduacao, Departamento de Pediatria, Faculdade de Medicina (FMUSP), Universidade de São Paulo, SP, Brasil. 4. Coordenadora do Comitê de Crise, Hospital das Clínicas, Faculdade de Medicina da Universidade de São Paulo (HC-FMUSP), São Paulo, SP, Brasil.
\end{abstract}

\section{SUMMARY}

On 11th March 2020, the World Health Organization (WHO) declared the COVID-19 a pandemic. The Obstetrics and Neonatology disciplines needed to be revised to suit the institutional need to expand intensive care beds to care for confirmed or suspected patients with COVID-19 in the state of São Paulo, following the recommendations of the Institutional Crisis Committee. Three different actions were needed: the structuring of teams and advanced medical post to attend COVID-19-free patients and those with suspect or confirmed COVID-19; elaborating the protocols from the delivery room throughout hospitalization. Some special considerations about breastfeeding and rooming-in were needed. The third action was the drafting of a protocol to admit infants from other hospitals with confirmed COVID-19 as the unit never admitted outpatients before.

KEYWORDS: Coronavirus Infections. Intensive Care Units, Neonatal. Breastfeeding. Delivery rooms.

\section{INTRODUCTION}

Since December 2019, cases of pneumonia have been reported in Wuhan, China caused by a new coronavirus, later called SARS-CoV-2 (Severe Acute Respiratory Syndrome by Coronavirus 2$)^{1}$. The disease called COVID-19 (Coronavirus Infectious Disease -2019) spread in China and, a few months later, it had reached more than 23 countries worldwide.
On March 11, 2020, the World Health Organization (WHO) declared a pandemic situation ${ }^{2}$. The Obstetrics and Neonatology disciplines needed to be revised to suit the institutional need to expand intensive care beds to care for confirmed or suspected patients with COVID-19 in the state of São Paulo, following the recommendations of the Institutional Crisis Committee. 
Structuring of teams and advanced medical post:

The first step consisted of discharging all patients eligible for discharge or transfer to other units able to attend them. Simultaneously, 14 beds at the Neonatal Intensive Care Unit (NICU) and 8 beds of intermediate care at the University Hospital were installed. The Neonatal Center sent equipment, materials, and supplies to facilitate the operation of the advanced medical post. Employees were also sent: doctors (13), nursing professionals (30), physiotherapists (6), a nutritionist (1), a social worker (1), and a psychologist (1).

Twelve doctors, thirty-two nursing professionals, six physiotherapists, a nutritionist, and a social worker remained to attend newborn (NB) babies, at the delivery room, of pregnant women with suspected or confirmed COVID and infants weighing up to $6 \mathrm{~kg}$ with confirmed COVID admitted at the Emergency Room (ER) or at another hospital.

The neonatology discipline revised its protocols and internal flows to adapt to the new demand.

\section{SERVICE FLOWCHART FROM THE DELIVERY ROOM TO THE NICU}

Delivery room:

Considering that the disease affects individuals of all ages but seems to be less severe in children ${ }^{3}$ and that there has not yet been evidence of vertical transmission of the virus ${ }^{4,5}$, the discipline suggests that:

- all NB of pregnant women with a flu-like syndrome at the time of delivery or who have confirmation of COVID-19 are considered a suspected case for 14 days before delivery up to 28 days after it ${ }^{6}$.

- precautions should prevent the NB from becoming contaminated after birth and also prevent the contamination by health professionals, if there is manipulation of the airways ${ }^{7}$.

- in the delivery room, the most experienced pediatrician is responsible for resuscitation, following all recommendations regarding the use of PPE (disposable long-sleeve waterproof apron, gloves, hat, N95 or FP2 mask, and face shield).

- Skin-to-skin contact and breastfeeding in the first hour are suspended for the duration of the pandemic.

- HEPA filter (High-Efficiency Particular Air) between the mask to be attached to the NB, in case it is necessary to perform positive pressure ventilation (PPV) and the device employed (self-inflating balloon or manual T-shaped mechanical ventilator) ${ }^{7}$.

The NB will be transported in a transport incubator to the admission sector with the team in full attire.

\section{Procedures during Hospitalization:}

Since the beginning of the pandemic, the wing dedicated to rooming-in has been disabled for this purpose, making it impossible to keep the dyad together during hospitalization. Rooming-in is not contraindicated as long as the mother is able to remain in the infirmary and receives guidelines for cough etiquette, proper hand hygiene before handling the newborn, and wears a surgical mask that must be changed if wet or as instructed by the manufacturer. Puerperal women diagnosed with COVID-19 must stay in a private room, and the presence of a single, healthy companion without home contact with COVID-19 and under 60 years old is possible. There is no evidence of the presence of viruses in human milk to date. The benefits of breastfeeding outweigh the risks of SARS-CoV-2 transmission, and breastfeeding should be encouraged and encouraged ${ }^{6,8,9}$.

However, just as the neonatology team was divided to meet the needs for assistance at the Clinics Hospital and at the University Hospital outpost, the obstetrics team suffered the same division. Thus, for operational reasons, rooming-in could not be maintained, and the separation of the dyad is necessary. The admission of mothers and newborns in different places is a consequence that was not foreseen at first and that may have uncalculable repercussions in breastfeeding ${ }^{10}$.

All newborns born in the complex are therefore admitted to the Neonatal Intensive Care Center and separated according to the mother's diagnosis.

In Room 1, there are the newborns whose mothers have confirmed COVID-19, with their own air conditioning system, with double filter. The search for SARS-CoV-2 by RT-PCR (Reverse Transcription Polymerase Chain Reaction) in tracheal secretion (when intubated) or oropharyngeal swab will be performed with 48 hours of life.

In Rooms 4 and 5, the NB whose mothers await the results of the SARS-CoV-2 survey are admitted, with a separate air conditioning system. When the diagnosis is ruled out, if the NB is more than 48 hours 
old and is healthy and all routines are complete, they will be discharged home or if there is any need for hospitalization, they will be sent to a COVID free unit. SARS-CoV-2 research will not be performed on these patients

NB should receive the care indicated for gestational age and the most prevalent diseases of this age group, and the treatment chosen must respect these characteristics. In view of the lack of knowledge of characteristic symptoms of COVID-19 in the neonatal period, newborns must be evaluated and treated as usual without running the risk of committing iatrogenesis. Respiratory insufficiencies in the neonatal period can be due to many diseases such as meconium aspiration syndrome, transient tachypnea, respiratory distress syndrome, and pneumonia for example. It is important that the neonatologist indicates the treatment that they consider most appropriate for their patient ${ }^{11}$. At the same time, it is equally important to be alert to unusual signs and symptoms that the NB may experience.

If there is confirmation of the presence of SARSCoV-2 in the newborn, they will be transferred to a room with anteroom in contact isolation and aerosols or droplets depending on the severity of the condition or procedure to be performed.

The Neonatal Center is located inside a hospital dedicated to the care of patients with COVID-19 exclusively, so there is no transit of visits in the building, and the circulation of mothers with SARS-CoV-2 in the unit is not possible due to the risk contamination of newborns whose research is negative. Daily, the medical team contacts the mothers and/or another responsible person designated by the family to give news about the newborn.

\section{Outpatients diagnosed with COVID-19}

Community transmission of SARS-CoV-2 in the municipality of São Paulo has already been identified and, since March 24, 2020, quarantine has been instituted with the closure of activities not considered essential, suspension of classes, and social distancing. Despite the imposition of these measures, new cases have still emerged. The horizontal transmission maintenance reflects some social aspects not calculated. Some families, despite respecting the recommendations, live in crowded small houses with many people sharing the environment, facilitating the contamination of everyone including newborns.
External NB who have been admitted to the NICU so far had fever, tachypnea, nasal obstruction, usually with good saturation. The respiratory condition was not always the most relevant, and fever without source (FWS) was a possible diagnosis on arrival at the hospital.

\section{Patients with flu syndrome:}

- collect viral panel to search for respiratory syncytial virus, H1N1, influenza A

- RT-PCR (Reverse-transcriptase-polymerase chain reaction) for SARS-CoV-2

- X-ray (X-ray) of the chest

- Chest ultrasound (US).

- Discuss chest CT indication

- Initially, the patient should receive oseltamivir until the etiology of the viral condition is established. If the H1N1 test is negative, the medication may be stopped.

Fever without source (FWS) with identification of caregiver or resident with flu syndrome or diagnosis of COVID-19:

- collect viral panel to search for respiratory syncytial virus, H1N1, influenza A

- RT-PCR for SARS-CoV-2

- Blood count with platelets

- C-Reactive Protein (PCR)

- Blood culture (HMC)

- Urine 1

- Uroculture (URC)

- Liquor.

- Chest X-ray

- Assess need for chest US

- If there are no respiratory symptoms suggestive of flu, it is not necessary to introduce oseltamivir.

- Introduction of antibiotics to cover FWS/late neonatal sepsis according to the patient's age. Antibiotic therapy should be suspended if a bacterial infection is ruled out.

In both situations, if there is a diagnostic confirmation of COVID-19, collect creatine phosphokinase (CPK), troponin, d-dimer, and fibrinogen levels.

The chest tomography will be performed during hospitalization according to clinical evaluation, and new tests will be indicated according to the patient's clinical evolution. The NB will be hospitalized at least until the seventh day after the onset of symptoms, and that period may be extended for up to 14 days 
depending on the evolution. During the stay in the hospital, visits from the asymptomatic mother will be allowed in the afternoon and the human milk collection room can perform the extraction of breast milk to offer to the child during her absence. The collection room is equipped and prepared according to the guidelines of the Ministry of Health ${ }^{11,12}$.

\section{Conflicts of Interest}

The authors declare no conflicts of interest.

\section{RESUMO}

PALAVRAS-CHAVE: Infecções por Coronavirus. Unidades de Terapia Intensiva Neonatal. Aleitamento Materno. Salas de Parto.

\section{REFERENCES}

1. Zhu N, Zhang D, Wang W, Li X, Yang B, Song J, et al; China Novel Coronavirus Investigating and Research Team. A novel coronavirus from patients with pneumonia in China, 2019. N Engl J Med. 2020;382(8):727-33.

2. Cucinotta D, Vanelli M. WHO declares COVID-19 a pandemic. Acta Biomed. 2020;91(1):157-60

3. Dong Y, Mo X, Hu Y, Qi X, Jiang F, Jiang Z, et al. Epidemiology of COVID-19 among children in China. Pediatrics. 2020;e20200702.

4. Chen H, Guo J, Wang C, Luo F, Yu X, Zhang W, et al. Clinical characteristics and intrauterine vertical transmission potential of COVID-19 infection in nine pregnant women: a retrospective review of medical records. Lancet. 2020;395(10226):809-15

5. Zeng L, Xia S, Yuan W, Yan K, Xiao F, Shao J, et al. Neonatal early-onset infection with SARS-CoV-2 in 33 neonates born to mothers with COVID-19 in Wuhan, China. JAMA Pediatr. 2020;e200878. doi: 10.1001/ jamapediatrics.2020.0878.

6. Center for Disease Control and Prevention (CDC). Interim considerations for infection prevention and control of coronavirus disease 2019 (COVID-19) in inpatient obstetric healthcare settings. [cited 2020 May 3]. Available from: https://www.cdc.gov/coronavirus/2019-

7. Sociedade Brasileira de Pediatria. Recomendações para assistência ao recém-nascido na sala de parto de mãe com COVID-19 suspeita ou confirmada: atualização. [cited 2020 May 3]. Available from: https://www.
sbp.com.br/fileadmin/user_upload/22422d-NAlerta-Assist_RN_SalaParto_de_mae_com_COVID-19.pdf

8. Royal College of Obstetricians and Gynaecologists. Coronavirus (COVID-19) infection and pregnancy. Information for healthcare professionals. Version 1: Published Monday 9 March, 2020. [cited 2020 May 3]. Available from: https://www.rcog.org.uk/globalassets/documents/guidelines/2020-0513-coronavirus-covid-19-infection-in-pregnancy.pdf

9. BRASIL. Ministério da Saúde. Nota Técnica No 6/2020-COCAM/ CGCIVI/DAPES/SAPS/MS. Atenção à saúde do recém-nascido no contexto da infecção do novo coronavírus (SARS-CoV-2). Brasília: Ministério da Saúde; 2020. [cited 2020 May 3]. Available from: https://sei.saude. gov.br/sei/

10. De Luca D. Managing neonates with respiratory failure due to SARS-CoV-2. Lancet Child Adolesc Health. 2020;4(4):e8.

11. BRASIL. Ministério da Saúde. Fundação Oswaldo Cruz. Rede Brasileira de Bancos de Leite Humano. Recomendação técnica No.01/20.170320. Covid19 e amamentação. [cited 2020 May 3]. Available from: https://rblh.fiocruz. br/covid-19-e-amamentacao-recomendacao-n0120170320

12. Mariani Neto C. Nótula complementar sobre COVID-19 e aleitamento materno. FEBRASGO. [cited 2020 Mar 11]. Available from: https://www.febrasgo.org.br/pt/noticias/ item/949-notula-complementar-sobre-covid-19-e-aleitamento-materno 